\title{
Cognitive function, drusen, and age-related macular degeneration: a cross-sectional study
}

H Lindekleiv ${ }^{1}, \mathrm{MG} \mathrm{Erke}^{1,2}, \mathrm{G}$ Bertelsen ${ }^{1,2}$, T Peto ${ }^{3}$, KA Arntzen ${ }^{1,2}$, H Schirmer ${ }^{1,2}$, T Wilsgaard ${ }^{1}$, EB Mathiesen ${ }^{1,2}$ and I Njølstad ${ }^{1}$

\begin{abstract}
Purpose To examine the cross-sectional relationship between drusen, late age-related macular degeneration (AMD), and cognitive function.

Methods We included 2149 stroke-free participants from the population-based Tromsø Study in Norway. Retinal photographs were graded for presence of drusen and AMD. Cognitive function was assessed using the verbal memory test (short verbal memory), digit-symbol coding test (processing speed), and the tapping test (psychomotor tempo). We assessed the relationship between drusen, late AMD, and cognitive test scores, adjusted for potential confounders.
\end{abstract}

Results Late AMD was associated with decreased performance in the verbal memory test (standardized $\beta=-0.23,95 \%$ confidence interval (CI): -0.51 to -0.01 ). Intermediate and large drusen were associated with decreased performance in the digit-symbol coding test (standardized $\beta=-0.14$ and $-0.19,95 \%$ CIs: -0.23 to -0.05 and -0.29 to -0.09 , respectively). Participants with large drusen were more likely to have test scores in the lowest quartile of the digit-symbol coding test (odds ratio $(\mathrm{OR})=1.9,95 \% \mathrm{CI}: 1.1-3.5)$ and the tapping test $(\mathrm{OR}=1.6,95 \%$ CI: 1.0-2.6), but not in the verbal memory test $(\mathrm{OR}=1.0,95 \% \mathrm{CI}$ : 0.6-1.6).

Conclusions The findings suggest a relationship between drusen deposition and reduced cognitive function. Although the relationships between drusen, late $A M D$, and the cognitive test results varied in strength and significance across the types of cognitive test, and may partly have been caused by residual confounding, it is not unlikely that a genuine but weaker relationship exists between drusen deposition and cognitive decline.

Eye (2013) 27, 1281-1287; doi:10.1038/eye.2013.181; published online 23 August 2013

Keywords: drusen; AMD; cognitive function

\section{Introduction}

Age-related macular degeneration (AMD) is an important cause of visual impairment and blindness in the elderly. ${ }^{1}$ Although the pathophysiology of AMD is not fully understood, presence of drusen is considered a component of the early stages of AMD. Drusen are accumulations of extracellular material traditionally found in Bruch's membrane, but may occur internal to the retinal pigment epithelium. ${ }^{2}$ Bruch's membrane separates the retinal pigment epithelium from the capillary lamina of the choroid and is important for exchange of nutrients, oxygen, and waste products between the outer retina and the circulatory system. ${ }^{3,4}$ Late stages of AMD include geographic atrophy and choroidal neovascularization.

Studies suggest that AMD is associated with both reduced cognitive function and Alzheimer's disease. ${ }^{5-8}$ Cognitive function is the mental processes by which the brain acquires, processes, and acts upon information. Decline in cognitive function may be seen as a continuum from normal ageing to mild cognitive impairment and dementia. On the basis of similarities in the histopathology of retinal drusen and Alzheimer's disease, a common pathogenesis has been hypothesized. In both diseases, extracellular accumulation of lipids, beta-amyloid, and waste products are found. ${ }^{9-11}$ Further, suggested risk factors for both disorders include old age, smoking,
${ }^{1}$ Faculty of Health Sciences, University of Tromsø, Tromsø, Norway

${ }^{2}$ University Hospital of North Norway, Tromsø, Norway

${ }^{3} \mathrm{NIHR}$ Biomedical Research Centre at Moorfields Eye Hospital and University College London Institute of Ophthalmology, London, UK

Correspondence:

$\mathrm{H}$ Lindekleiv, Department of Community Medicine,

Faculty of Health Sciences, University of Tromsø, N-9037, Tromsø, Norway Tel: + 4797136866 ; Fax: +47 77644831 . E-mail: haakon.lindekleiv@ gmail.com

Received: 29 January 2013 Accepted in revised form: 20 July 2013 Published online: 23 August 2013 
hypercholesterolemia, hypertension, obesity, and atherosclerosis. However, population-based studies on the relationship between AMD and cognitive functions are few and have only reported weak associations. ${ }^{5-8}$ Further studies are warranted to examine the relationship between cognitive function and AMD.

The present study aimed to examine the relationship between AMD and cognitive function in a population-based cross-sectional study.

\section{Materials and methods}

\section{Study populations}

The Troms $\varnothing$ Study is a prospective, population-based cohort study. The study is conducted in the municipality of Troms $\varnothing$, Troms, Norway, and comprises a homogenous, Caucasian population. The sixth survey of the Troms $\varnothing$ study was conducted in $2007 / 2008 .{ }^{12}$ All residents in the municipality of Troms $\varnothing$ aged 40-42 and $60-87$ years, $10-40 \%$ random samples of birth cohorts aged 30-59 years, and if not already included in the two groups above, persons who had attended the second visit of Troms $\varnothing 4$ and were aged $<75$ years in 1994, were invited.

The sixth survey included a second visit with more extensive examinations, including retinal photography (The Troms $\varnothing$ Eye Study) and cognitive tests. The eligible population for the second visit were first-visit participants aged 50-62 or 75-84 years, a $20 \%$ random sample of men and women aged 63-74 years, and participants, if not already included in the two groups above, who had attended the extended visit of the fourth Tromsø survey and were aged $<75$ years in 1994 . Of 11484 eligible participants, 7307 (64\%) attended. Participants aged $\geq 65$ years $(n=3025)$ were included in the present study.

We excluded 152 participants with prior stroke because cognitive impairment is frequent after stroke. ${ }^{13}$ We further excluded 442 participants who did not undergo cognitive tests because of logistic reasons and 282 participants without retinal photographs because of lack of consent or logistic reasons. This left 2149 participants for analysis.

All participants gave informed, written consent. The Regional Ethics Committee approved the study.

\section{Data collection}

Information on body height and weight, systolic blood pressure, serum total cholesterol, smoking habits, alcohol consumption, depression, diabetes, education level, physical activity, and use of antihypertensive or antidepressant treatment was obtained from physical examination and self-reported questionnaires. Body mass index was calculated by dividing body weight (kilograms) with the square of height (meters). Blood pressure was measured by specially trained personnel, using an automatic device (Dinamap Vital Signs Monitor, Tampa, FL, USA). Hypertension was defined as systolic blood pressure $>140 \mathrm{~mm} \mathrm{Hg}$, diastolic blood pressure $>90 \mathrm{~mm} \mathrm{Hg}$, or use of antihypertensive treatment. Nonfasting serum total cholesterol was analyzed using standard enzymatic methods at the Department of Clinical Chemistry, University Hospital of North Norway. On the basis of the self-reported questionnaires, the participants' daily smoking habits were classified as never (reference category), former, and current. Alcohol consumption was classified as abstinent, drinking $<1$ time per month (reference category), 1-4 times per month, and $>4$ times per month. Depression was defined as use of antidepressant medication or answering the question 'Have you in the last two weeks felt down/depressed?' in the upper two of the four categories (no, a little, a lot, or very much). Diabetes was defined as self-reported diabetes, use of anti-diabetic medication, or serum-glycated hemoglobin (HbA1c) $>6.5 \%$. The highest level of education attained was categorized as Primary school (reference category), Secondary school, or College/University. Physically active was defined as $\geq 1$ time exercise per week with sweating or out of breath or $\geq 2$ times exercise per week without sweating or out of breath. Corrected visual acuity was assessed using autorefractor according to Snellen. Impaired vision was defined as Snellen $\leq 0.32$.

Cognitive function was assessed using three cognitive tests. The 12-word memory test is a test of short verbal memory. Twelve nouns were shown written on a board and also pronounced one at a time. Participants had 2 min to recall the words. The digit-symbol coding test is a part of Wechsler adult intelligence scale and is a test of attention and processing speed. The test consists of rows containing small blank squares, each paired with a randomly assigned number from one to nine. Above these rows was a printed key that paired each number with a different nonsense symbol. Following a practice trial with the first seven numbers, participants were asked to consecutively fill in as many as possible of the blank spaces with the corresponding symbol over $90 \mathrm{~s}$. Participants were encouraged to perform the task as quickly and accurately as possibly. The tapping test is a test of psychomotor tempo. Participants were instructed to tap as many times as possible in $10 \mathrm{~s}$ with their index finger. The taps were performed on a computer that registered the number of taps. The task was repeated four times on both dominant hand and non-dominant hand. The mean of the average number of the three last repeats for each hand was used in the analyses. ${ }^{14}$ In order to make the cognitive test scores comparable, we transformed the score for each test into a standardized 
$z$-score (the difference between each test score minus the average score, divided by the SD of that score).

One of the authors (M.G.E.) graded retinal photographs for AMD on the basis of the International Classification and Grading System for AMD. ${ }^{15}$ Details on the grading procedure and quality control have been described previously. ${ }^{16,17}$ Photographs were graded for predominant phenotype determined by the most severe lesion present within the macula. Categories were late AMD (choroidal neovascularization or geographic atrophy), large drusen ( $>125 \mu \mathrm{m})$, intermediate drusen (63-125 $\mu \mathrm{m})$, and normal phenotype (no drusen or drusen $<63 \mu \mathrm{m}$ ). The grade from the more affected eye was assigned to each participant.

\section{Statistical analyses}

Descriptive statistics were calculated to characterize late-AMD cases and study participants.

The relationship between the four predominant phenotypes from the most affected eye and cognitive function was assessed by multivariable linear regression. We used the different cognitive tests as dependent variables and three indicator variables for the phenotype categories as independent variable, leaving participants with normal phenotype only as the reference group. Potential confounding factors (listed in Table 1) were entered into the models, and nonsignificant variables were dropped using backward elimination with a selection level of $<0.10$. Removal of nonsignificant variables did not change the estimated beta-coefficients for the graded phenotypes. $P$ for trend was calculated by entering the category variable 'dominant phenotype' as a continuous variable in the regression models. Posthoc regression diagnostics were performed to assess the assumptions underlying linear regression. Normal distribution of residuals was verified using histograms of residuals. Homoscedasticity was verified by visual inspection of scatter plots of the residuals on the predicted values. Multicollinearity was not of concern (variance inflation factor $<1.3$ for all variables).

Table 1 Characteristics of study participants: the Tromsø Study

\begin{tabular}{|c|c|c|c|c|c|}
\hline Variable & $\begin{array}{l}\text { Normal phenotype } \\
\qquad(\mathrm{n}=831)\end{array}$ & $\begin{array}{l}\text { Intermediate drusen } \\
\qquad(\mathrm{n}=732)\end{array}$ & $\begin{array}{l}\text { Large drusen } \\
\quad(\mathrm{n}=514)\end{array}$ & $\begin{array}{l}\text { Late } A M D \\
\quad(\mathrm{n}=76)\end{array}$ & $\begin{array}{l}\text { All participants } \\
\quad(\mathrm{n}=2149)\end{array}$ \\
\hline Female sex, $n(\%)$ & $443(54 \%)$ & $431(59 \%)$ & $312(61 \%)$ & $35(46 \%)$ & $1228(57 \%)$ \\
\hline Age, in years & $71.2 \pm 4.9$ & $71.6 \pm 5.0$ & $73.8 \pm 5.6$ & $76.9 \pm 5.3$ & $72.2 \pm 5.3$ \\
\hline Hypertension $^{\mathrm{a}}, n(\%)$ & $606(75 \%)$ & $542(74 \%)$ & $398(78 \%)$ & $69(91 \%)$ & $1629(76 \%)$ \\
\hline Diabetes $^{\mathrm{b}}, n(\%)$ & $82(10 \%)$ & $69(9 \%)$ & $49(10 \%)$ & $9(12 \%)$ & $213(10 \%)$ \\
\hline Depression $^{\mathrm{c}}, n(\%)$ & $48(6 \%)$ & $44(6 \%)$ & $36(7 \%)$ & $4(5 \%)$ & $134(6 \%)$ \\
\hline Physically active $^{\mathrm{d}}, n(\%)$ & $295(36 \%)$ & $245(33 \%)$ & $160(31 \%)$ & $17(22 \%)$ & $717(33 \%)$ \\
\hline Body mass index, in $\mathrm{kg} / \mathrm{m}^{2}$ & $27.2 \pm 4.1$ & $26.8 \pm 3.9$ & $27.1 \pm 4.4$ & $26.6 \pm 3.8$ & $27.0 \pm 4.1$ \\
\hline Total Cholesterol, in mmol/1 & $5.7 \pm 1.1$ & $5.7 \pm 1.1$ & $5.7 \pm 1.1$ & $5.6 \pm 3.8$ & $5.7 \pm 1.1$ \\
\hline \multicolumn{6}{|l|}{ Smoking habits, n (\%) } \\
\hline Never smoker & $297(37 \%)$ & $279(39 \%)$ & $198(39 \%)$ & $16(23 \%)$ & $795(38 \%)$ \\
\hline Former smoker & $382(48 \%)$ & $346(49 \%)$ & $236(47 \%)$ & $37(53 \%)$ & $1,007(48 \%)$ \\
\hline Daily smoker & $117(15 \%)$ & $89(13 \%)$ & $69(14 \%)$ & $17(24 \%)$ & $293(14 \%)$ \\
\hline \multicolumn{6}{|l|}{ Alcohol consumption, n (\%) } \\
\hline Abstinent & $144(18 \%)$ & $134(19 \%)$ & $132(27 \%)$ & $20(29 \%)$ & $433(21 \%)$ \\
\hline$\leq 1$ time per month & $286(36 \%)$ & $220(31 \%)$ & $183(37 \%)$ & $23(33 \%)$ & $717(34 \%)$ \\
\hline 2-4 times per month & $208(27 \%)$ & $227(32 \%)$ & $108(22 \%)$ & $18(26 \%)$ & $563(27 \%)$ \\
\hline$>4$ times per month & $150(19 \%)$ & $130(18 \%)$ & $72(14 \%)$ & $9(13 \%)$ & $364(18 \%)$ \\
\hline \multicolumn{6}{|c|}{ Highest level of education attained, $\mathrm{n}(\%)$} \\
\hline Primary school & $351(44 \%)$ & $328(46 \%)$ & $263(53 \%)$ & $40(54 \%)$ & $990(47 \%)$ \\
\hline Secondary school & $280(35 \%)$ & $208(29 \%)$ & $149(30 \%)$ & $23(31 \%)$ & $665(32 \%)$ \\
\hline College/University & $164(21 \%)$ & $171(25 \%)$ & $84(17 \%)$ & $11(15 \%)$ & $430(21 \%)$ \\
\hline Impaired vision ${ }^{\mathrm{e}}, n(\%)$ & $7(1 \%)$ & $8(1 \%)$ & $12(2 \%)$ & $16(23 \%)$ & $46(2 \%)$ \\
\hline
\end{tabular}

Abbreviation: AMD, age-related macular degeneration.

All values are means \pm SD unless otherwise indicated.

a Defined as: Systolic blood pressure $\geq 140 \mathrm{~mm} \mathrm{Hg}$, diastolic blood pressure $\geq 90 \mathrm{~mm} \mathrm{Hg}$, or use of antihypertensive treatment.

${ }^{\mathrm{b}}$ Self-reported diabetes, use of anti-diabetic medication, or serum glycated hemoglobin (HbA1c) $>6.5 \%$.

'Answering the question 'Have you in the last two weeks felt down/depressed?' in the upper two of the four categories ('a lot' or 'very much').

d $\geq 1$ times exercise per week with sweating or out of breath or $\geq 2$ times exercise per week without sweating or out of breath.

e Snellen $\leq 0.32$. 
No validated cutoff value for cognitive impairment exists for the cognitive tests. The results of each cognitive test were divided in quartiles, and a logistic regression model was fitted to calculate the adjusted odds ratio (OR) for having a test result in the lowest quartile compared with the highest quartile for the groups of predominant phenotype of the worst eye, treating participants with normal phenotype as the reference. Post hoc analyses found the models to fit the data well (Hosmer-Lemeshow goodness-of-fit; all $P$-values $>0.21$ ).

Data were analyzed using STATA software (Version 12.0, Stata Corp, TX, USA). All comparisons used a two-sided significance level of $5 \%$.

\section{Results}

A total of 2149 stroke-free participants $\geq 65$ years old were graded for AMD and attended at least one cognitive test (2141 attended the verbal memory test; 2046 attended the digit-symbol coding test; and 1993 attended the tapping test). In total, 76 participants had late AMD (54 neovascular AMD and 22 geographic atrophy), 514 participants had large drusen, 732 participants had intermediate drusen, 813 participants had normal phenotype, and 14 participants had ungradable retinal photographs. Baseline characteristics are provided in Table 1.

Compared with those included in the study, the 876 excluded participants were older (73.2 vs 72.2 years, $P<0.01)$, less physically active (25 vs 33\%, $P<0.01$ ), and more likely to have diabetes ( 15 vs $10 \%, P<0.01$ ), but did not differ with respect to the other baseline characteristics (data not shown).

The association between cognitive test scores, drusen, and late AMD, adjusted for possible confounders, are shown in Table 2. We found significant decreased score in the digit-symbol coding test for participants with large drusen (standardized $\beta=-0.14,95 \%$ confidence interval (CI): -0.23 to -0.05 ) and for participants with intermediate drusen (standardized $\beta=-0.19,95 \% \mathrm{CI}$ : -0.29 to -0.09 ). Late AMD was associated with significant decreased score in the word memory test (standardized $\beta=-0.23,95 \% \mathrm{CI}$ : -0.51 to -0.01 ).

The ORs for poor (lowest quartile) $v s$ the best (highest quartile) results on the cognitive tests, adjusted for potential confounders, are shown in Table 3. The ORs for poor performance in the digit-symbol coding test and tapping test were significantly higher for participants with large drusen, compared with participants with normal phenotype.

\section{Discussion}

We found that participants with late AMD had lower scores in the verbal memory test, and participants with large and intermediate drusen had lower scores in the digit-symbol coding test than participants with normal phenotype.

The cross-sectional relationship between AMD and cognitive function has been examined in four previous population-based cross-sectional studies (Table 4). ${ }^{5-8}$

Table 2 Association between standardized ${ }^{a}$ cognitive test scores, drusen, and late AMD, adjusted for potential confounders: the Troms $\varnothing$ Study

\begin{tabular}{|c|c|c|c|c|c|c|c|}
\hline & \multirow[t]{2}{*}{ Cases $^{\mathrm{b}}$} & \multicolumn{2}{|c|}{ Verbal memory test } & \multicolumn{2}{|c|}{ Digit-symbol coding test } & \multicolumn{2}{|c|}{ Tapping test } \\
\hline & & $\beta$ & $95 \% C I$ & $\beta$ & $95 \% \mathrm{CI}$ & $\beta$ & $95 \% \mathrm{CI}$ \\
\hline \multicolumn{8}{|l|}{ Age-adjusted } \\
\hline Normal phenotype & 813 & 0.0 & Reference & 0.0 & Reference & 0.0 & Reference \\
\hline Intermediate drusen & 732 & -0.01 & -0.10 to 0.09 & -0.09 & -0.18 to 0.00 & -0.06 & -0.17 to 0.04 \\
\hline Large drusen & 514 & -0.08 & -0.19 to 0.02 & -0.19 & -0.29 to -0.08 & -0.04 & -0.24 to -0.01 \\
\hline Late AMD & 76 & -0.26 & -0.49 to -0.03 & -0.23 & -0.47 to 0.01 & -0.05 & -0.33 to 0.19 \\
\hline$P$ for trend & & & $P=0.03$ & & $P<0.001$ & & $P=0.05$ \\
\hline \multicolumn{8}{|l|}{ Multivariable adjusted } \\
\hline Normal phenotype & 813 & 0.0 & Reference & 0.0 & Reference & 0.0 & Reference \\
\hline Intermediate drusen & 732 & -0.02 & -0.12 to 0.06 & -0.14 & -0.23 to -0.05 & -0.06 & -0.16 to 0.03 \\
\hline Large drusen & 514 & -0.06 & -0.16 to 0.04 & -0.19 & -0.29 to -0.09 & -0.08 & -0.19 to 0.03 \\
\hline Late AMD & 76 & -0.23 & -0.51 to -0.01 & -0.14 & -0.38 to 0.09 & -0.05 & -0.30 to 0.21 \\
\hline$P$ for trend & & & $P=0.07$ & & $P<0.001$ & & $P=0.15$ \\
\hline
\end{tabular}

Abbreviations: AMD, age-related macular degeneration; CI, confidence interval.

${ }^{\text {a }}$ One unit of cognitive score $=$ one SD.

${ }^{\mathrm{b}}$ Number of cases analyzed for each cognitive test varied because of missing observations.

${ }^{c}$ The verbal memory test adjusted for age, sex, depression, alcohol consumption, and the highest level of education attained; the digit-symbol coding test adjusted for age, sex, diabetes, smoking, alcohol consumption, and the highest level of education attained; the tapping test adjusted for age, sex, diabetes, physical activity, alcohol consumption, and the highest level of education attained. 
Table 3 Adjusted $^{\text {a }}$ OR for poor (lowest quartile) vs the best (highest quartile) performance on cognitive tests in subjects with late AMD or drusen: the Tromsø Eye Study

\begin{tabular}{|c|c|c|c|c|c|c|c|}
\hline & \multirow[t]{2}{*}{ Cases } & \multicolumn{2}{|c|}{ Verbal memory test } & \multicolumn{2}{|c|}{ Digit-symbol coding test } & \multicolumn{2}{|c|}{ Tapping test } \\
\hline & & OR & $95 \% C I$ & OR & $95 \% C I$ & OR & $95 \% C I$ \\
\hline Normal phenotype & 813 & 1.0 & Reference & 1.0 & Reference & 1.0 & Reference \\
\hline Intermediate drusen & 732 & 1.1 & $0.8-1.8$ & 1.6 & $1.0-2.6$ & 1.5 & $1.0-2.4$ \\
\hline Large drusen & 514 & 1.0 & $0.6-1.6$ & 1.9 & $1.1-3.5$ & 1.6 & $1.0-2.6$ \\
\hline Late AMD & 76 & 2.0 & $0.6-6.4$ & 2.0 & $0.3-15.7$ & 2.2 & $0.7-7.1$ \\
\hline$p$ for trend & & & $P=0.62$ & & $P=0.02$ & & $P=0.01$ \\
\hline
\end{tabular}

Abbreviations: AMD, CI, confidence interval; OR, odds ratio.

a The verbal memory test adjusted for age, sex, alcohol consumption, systolic blood pressure, and the highest level of education attained; the digit-symbol coding test adjusted for age, sex, depression, alcohol consumption, and the highest level of education attained; the tapping test adjusted for age, sex, total cholesterol, physical activity, alcohol consumption, and the highest level of education attained.

Although these studies used different cognitive tests ranging from crude global tests such as the mini-mental examination (MMS-E) to more sensitive tests such as the digit-symbol substitution test (DSST), the results indicate a relationship between poorer cognitive test results and AMD. However, the findings are weak and not consistent across different cognitive tests and across different studies. This inconsistency is also observed in our study. We found that late AMD was associated with lower score in the verbal memory test (short verbal memory), but not in the digit-symbol coding test (processing speed), and the tapping test (psychomotor tempo). One populationbased prospective cohort study has examined AMD as a predictor of incident Alzheimer's disease. ${ }^{8}$ In 1403 participants from the Rotterdam Study, no relationship between AMD and incident Alzheimer's disease was observed after adjustment for confounding factors (relative risk 1.5, 95\% CI: 0.6-3.5, adjusted for age, sex, smoking, and ankle brachial index).

The present study adds to the literature by examining the relationship between drusen, late AMD, and cognitive tests that are more sensitive to the early stages of dementia than global tests such as the mini-mental status examination. ${ }^{18}$ To our knowledge, the study includes the largest number of late AMD cases so far published from population-based cross-sectional studies. However, both previous studies and the present study were based on small samples of late AMD and may therefore lack the statistical power to detect weak or moderate relationships. It is also possible that significant associations may have been caused by residual confounding. For instance, as both AMD and cognitive decline are strongly age-related, it is difficult to know whether the effect of age can be fully adjusted for in the statistical models. Unfortunately, the low number of late AMD cases precluded age-stratified analyses.

The potential relationship between drusen deposition and cognitive decline indicates that drusen deposition might become a surrogate marker of cognitive decline.
More biomarkers for dementia and Alzheimer's disease are highly warranted, as the current biomarkers of dementia require invasive diagnostic procedures such as lumbar punction, and expensive neuroimaging such as magnetic resonance imaging and positron emission tomography. In contrast, retinal photography is both a non-invasive and relatively cheap procedure. Future studies of the relationship between AMD and cognitive disease should be conducted using pooled data from several prospective cohorts, as the sample size of each cohort is relatively small. In order to facilitate comparison of results, similar cognitive tests should be adopted in epidemiologic studies of eye disease. Further, there is a need for prospective cohort studies, as no causal inference between AMD and cognitive disease can be drawn from cross-sectional data. Such prospective studies could establish whether drusen and AMD are independent markers of dementia, and whether drusen and AMD have an added value in the diagnosis of dementia.

Strengths of the present study include a large, population-based sample, the use of sensitive cognitive tests feasible in a population screening setting, and the adjustment for visual acuity and potential confounders. The digit-symbol coding test is highly sensitive to cognitive impairment and is one of the first tests that reveals declining test scores during preclinical dementia. ${ }^{18}$ In comparison, global tests such as the minimental status examination are less likely to detect early dementia. ${ }^{18}$ The study has some limitations. First, the cross-sectional design precludes definite conclusions on the association between AMD and cognitive impairment. Second, selection bias may exist. Compared with those included, excluded participants were older, less physically active, and more likely to have diabetes. Further, the attendance rate is likely to be lower among persons with dementia. Third, ApoE status was not analyzed as data were available for $<10 \%$ of the participants. Fourth, the low number of late AMD cases 
Table 4 Existing population-based cross-sectional studies of cognitive function and AMD

\begin{tabular}{llll}
\hline Study $\begin{array}{c}\text { AMD } \\
\text { cases }\end{array}$ & Participants Design & Tests Main findings \\
Early Late & & &
\end{tabular}

Early $A M D$

Cardiovascular Health $\quad 324 \quad 27 \quad 2088$

Study ${ }^{5}$

Atherosclerosis Risk $\quad 448 \quad 12 \quad 9286$ in Communities ${ }^{6}$
Cross-sectional DSST MMS-E

Cross-sectional WFT

DSST
Participants with a score in the lowest quartile on the DSST were more likely to have early AMD than persons with higher scores (OR 1.38, 95\% CI: 1.03-1.85, controlled for confounders).

Participants with a WFT score in the lowest 10th percentile were more likely to have early AMD (OR $=1.6,95 \%$ CI: $1.1-2.2$, adjusted for confounders).

No relationship between late AMD and cognitive test scores

Late $A M D$

\begin{tabular}{|c|c|c|c|c|c|c|}
\hline Blue Mountains Study ${ }^{7}$ & 273 & 50 & 3509 & Cross-sectional & MMS-E & $\begin{array}{l}\text { Late AMD was associated with a low-normal score }(24-27) \text { on } \\
\text { the MMS-E }(\mathrm{OR}=2.2,95 \% \mathrm{CI}: 1.1-5.0) \text {. }\end{array}$ \\
\hline $\begin{array}{l}\text { Singapore Malay } \\
\text { Eye Study }\end{array}$ & - & 32 & 1179 & Cross-sectional & AMT & $\begin{array}{l}\text { No relationship between low score }(<7) \text { on the AMT and late } \\
\text { AMD was observed }(\mathrm{OR}=1.36,95 \% \mathrm{CI} \text { : } 0.35-5.45) \text {. }\end{array}$ \\
\hline Troms $\varnothing$ study & - & 76 & 2149 & Cross-sectional & $\begin{array}{l}\text { VMT } \\
\text { DSCT } \\
\text { Tap test }\end{array}$ & $\begin{array}{l}\text { Late AMD was associated with decreased performance in the verbal } \\
\text { memory test (standardized } \beta=-0.23,95 \% \mathrm{CI}:-0.51 \text { to }-0.01 \text { ). } \\
\text { No relationship between late AMD and other cognitive tests }\end{array}$ \\
\hline
\end{tabular}

Abbreviations: AMT, abbreviated mental test; CI, confidence interval; DSST, digit-symbol substitution test; DWRT, delayed word recall test; MMS-E, mini-mental status examination; OR, odds ratio; RR, relative risk; VMT, verbal memory test; WFT, word fluency test.

prevented evaluation of late AMD subtypes. Fifth, there is no validated cutoff score for the cognitive tests to classify individuals as cognitive impaired. Finally, the external validity refers to a Caucasian population and may not be generalizable to other ethnic groups.

In conclusion, the findings suggest a relationship between drusen deposition and reduced cognitive function. Although the relationships between drusen, late $\mathrm{AMD}$, and the cognitive test results varied in strength and significance across types of cognitive test, and may partly have been caused by residual confounding, it is not unlikely that a genuine but weaker relationship exists.

\section{Summary}

What was known before

- Studies suggest that AMD is associated with impaired cognitive function and dementia.

What this study adds

- The present study adds to the limited literature suggesting a relationship between drusen, AMD, and reduced cognitive function.

\section{Conflict of interest}

The authors declare no conflict of interest.

\section{Acknowledgements}

This study was supported by Grants from Simon Fougner Hartmanns familiefond, the Northern Norway Regional Health Authority (SFP897-09), the Norwegian
Research Council (176939/V40). Tunde Peto was supported by grants from the NIHR, UK.

\section{References}

1 Klaver CC, Wolfs RC, Vingerling JR, Hofman A, de Jong PT. Age-specific prevalence and causes of blindness and visual impairment in an older population: the Rotterdam Study. Arch Ophthalmologica 1998; 116(5): 653-658.

2 Zweifel SA, Spaide RF, Curcio CA, Malek G, Imamura Y. Reticular pseudodrusen are subretinal drusenoid deposits. Ophthalmology 2010; 117(2): 303-312.

3 Curcio CA, Millican CL, Bailey T, Kruth HS. Accumulation of cholesterol with age in human Bruch's membrane. Inves Ophthalmol Visual Sci 2001; 42(1): 265-274.

4 Booij JC, Baas DC, Beisekeeva J, TGMF Gorgels, Bergen AAB. The dynamic nature of Bruch's membrane. Prog Retin Eye Res 2010; 29(1): 1-18.

5 Baker ML, Wang JJ, Rogers S, Klein R, Kuller LH, Larsen EK et al. Early age-related macular degeneration, cognitive function, and dementia: the Cardiovascular Health Study. Arch Ophthalmol 2009; 127(5): 667-673.

6 Wong TY, Klein R, Nieto FJ, Moraes SA, Mosley TH, Couper DJ et al. Is early age-related maculopathy related to cognitive function? The Atherosclerosis Risk in Communities Study. Am J Ophthalmol 2002; 134(6): 828-835.

7 Pham TQ, Kifley A, Mitchell P, Wang JJ. Relation of age-related macular degeneration and cognitive impairment in an older population. Gerontology 2006; 52(6): 353-358.

8 Klaver CC, Ott A, Hofman A, Assink JJ, Breteler MM, de Jong PT. Is age-related maculopathy associated with Alzheimer's Disease? The Rotterdam Study. Am J Epidemiol 1999; 150(9): 963-968.

9 Anderson DH, Talaga KC, Rivest AJ, Barron E, Hageman GS, Johnson LV. Characterization of beta amyloid assemblies in drusen: the deposits associated with aging and age-related macular degeneration. Exp Eye Res 2004; 78(2): 243-256.

10 Mullins RF, Russel SR, Anderson DH, Hageman GS. Drusen associated with aging and age-related macular degeneration 
contain proteins common to extracellular deposits associated with atherosclerosis, elastosis, amyloidosis, and dense deposit disease. FASEB J 2000; 14(7): 835-846.

11 Braak H, Braak E. Neuropathological stageing of Alzheimerrelated changes. Acta Neuropathol 1991; 82(4): 239-259.

12 Jacobsen BK, Eggen AE, Mathiesen EB, Wilsgaard T, Njølstad I. Cohort profile: The Tromso Study. Int J Epidemiol 2012; 41(4): 961-967.

13 Tatemichi TK, Desmond DW, Stern Y, Paik M, Sano M, Bagiella E. Cognitive impairment after stroke: frequency, patterns, and relationship to functional abilities. J Neurol Neurosurg Psychiatry 1994; 57(2): 202-207.

14 Arntzen KA, Schirmer H, Wilsgaard T, Mathiesen EB. Moderate wine consumption is associated with better cognitive test results: a 7 year follow up of 5033 subjects in the Tromsø Study. Acta Neurol Scand Suppl 2010; 190: 23-29.

15 Bird AC, Bressler NM, Bressler SB, Chisholm IH, Coscas G, Davis MD et al. An international classification and grading system for age-related maculopathy and age-related macular degeneration. The International ARM Epidemiological Study Group. Surv Ophthalmol 1995; 39(5): 367-374.

16 Bertelsen G, Erke MG, von Hanno T, Mathiesen EB, Peto T, Sjölie AK et al. The Tromsø Eye Study: study design, methodology and results on visual acuity and refractive errors. Acta Ophthalmologica 2012, Epub ahead of print 11 September 2010; doi:10.1111/j.1755-3768.2012.02511.x.

17 Erke MG, Bertelsen G, Peto T, Sjölie AK, Lindekleiv H, Njølstad I. Prevalence of age-related macular degeneration in elderly Caucasians: the Tromsø Eye Study. Ophthalmology 2012; 119(9): 1737-1743.

18 Fabrigoule C, Rouch I, Taberly A, Letenneur L, Commenges D, Mazaux JM et al. Cognitive process in preclinical phase of dementia. Brain 1998; 121(Pt 1): 135-141. 